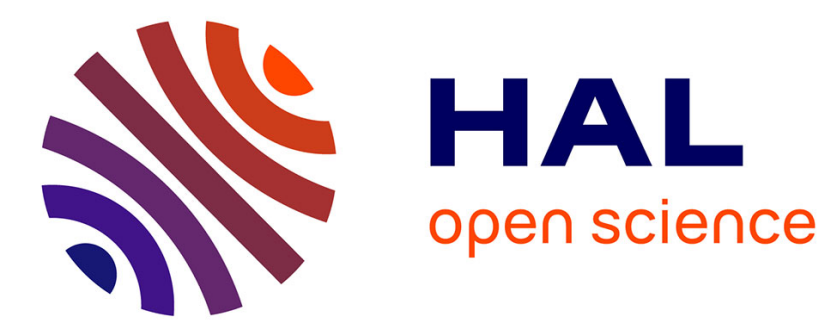

\title{
Les modifications de l'offre de transport: des effets automatiques à l'innovation socio-technique. L'exemple de la grande vitesse
}

Olivier Klein

\section{- To cite this version:}

Olivier Klein. Les modifications de l'offre de transport: des effets automatiques à l'innovation sociotechnique. L'exemple de la grande vitesse. Espaces et sociétés (Paris, France), 1998, tome 1 (95), pp. 95-126. halshs-00144735

\author{
HAL Id: halshs-00144735 \\ https://shs.hal.science/halshs-00144735
}

Submitted on 24 Jul 2012

HAL is a multi-disciplinary open access archive for the deposit and dissemination of scientific research documents, whether they are published or not. The documents may come from teaching and research institutions in France or abroad, or from public or private research centers.
L'archive ouverte pluridisciplinaire HAL, est destinée au dépôt et à la diffusion de documents scientifiques de niveau recherche, publiés ou non, émanant des établissements d'enseignement et de recherche français ou étrangers, des laboratoires publics ou privés. 


\section{LES MODIFICATIONS DE L'OFFRE DE TRANSPORT : DES EFFETS AUTOMATIQUES A L'INNOVATION SOCIO-TECHNIQUE L'EXEMPLE DE LA GRANDE VITESSE}

Article publié dans Espaces et Sociétés, n95, pp.95-126.

Olivier KLEIN

enseignant-chercheur

Ecole Nationale des Travaux Publics de l'Etat

Laboratoire d'Economie des Transports

\author{
rue Maurice Audin \\ 69518 Vaulx-en-Velin cedex \\ France \\ tel : (33) 0472047718 \\ fax : (33) 0472047092 \\ e-mail : olivier.klein@entpe.fr
}

\section{INTRODUCTION}

Les routes et les voies ferrées constituent l'archétype de l'infrastructure. Par leur réalité physique, leur emprise au sol et leur inscription dans le paysage, elles contribuent à dessiner la ville. Leur fonctionnement, les flux qu'elles génèrent ou qu'elles concentrent, constituent un second aspect à travers lequel les infrastructures donnent sa physionomie au tissu urbain. Nous voudrions dans cet article aborder la problématique "infrastructures et formes urbaines" d'un troisième point de vue, en considérant les activités humaines dont la réalisation amène l'usage d'un moyen de transport. Il s'agit, au-delà du déplacement lui-même qui n'est jamais un but en soi, de replacer le transport dans le fonctionnement économique et social de la cité.

Cette façon particulière d'interroger le système de transport impose tout d'abord un petit préalable théorique. Il s'agit d'adopter un cadre conceptuel qui permette de concevoir les relations entre transport et société. La disposition d'une offre de transport détermine-t-elle la réalisation de telle ou telle activité ? Ce sera l'objet de la première partie de ce texte que de faire le bilan des représentations qui ont prévalu en la matière et de construire une grille de lecture pertinente au regard des connaissances actuelles. On verra que l'on s'éloigne peu à peu d'une relation de type causal pour adopter un point de vue embrassant plus largement les évolutions de la société.

En se référant à la grille de lecture ainsi construite, il conviendra ensuite d'aborder la question des déplacements et des activités urbaines à partir d'observations concrètes. La seconde partie de cet article précisera de ce point de vue quelques tendances lourdes qui constituent l'environnement général contemporain des modifications d'offre de transport. La troisième partie détaillera l'exemple de quatre agglomérations dont les relations avec Paris ont été modifiées à la suite de la mise en service du TGV. Elle en précisera le contexte spécifique et sera en partie fondée sur les résultats de mobilité spécifiques destinées justement à préciser les motifs détaillés de déplacement de voyageurs à longue distance et à mesurer leur évolution à la faveur de la mise en service d'une desserte ferroviaire à grande vitesse.

Afin de ne pas trop étendre le propos, on se limitera dans cet article à l'observation des voyages à motif professionnel, plus directement en rapport avec les activités économiques. Les exemples évoqués illustreront le fait que les caractéristiques de l'offre de transport, les évolutions sociales et la conjoncture d'une époque, les spécificités de telle ou telle unité urbaine et les comportements de déplacement des différentes catégories de population forment un ensemble dont l’appréhension globale est nécessaire pour donner un sens aux évolutions concrètes constatées. 


\section{TRANSPORT, ECONOMIE ET SOCIETE : EVOLUTION DES REPRESENTATIONS}

Les représentations de la place de l'offre de transport dans l'économie et la société ont évolué. On peut distinguer trois grandes étapes : partant d'une vision parfaitement déterministe - l'offre de transport dispensatrice de richesse (1.1), on observe ensuite une première tentative pour se libérer de ce mécanicisme trop pesant - l'offre de transport à valoriser par les acteurs (1.2). C'est en essayant de tirer parti des lacunes de ces démarches que nous proposerons d'adopter un cadre conceptuel plus contemporain (1.3). Il n'y a pas, d'une étape à l'autre, de chronologie précise, quand bien même elles sont présentées ici dans leur ordre d'apparition. Il n'y a surtout pas remplacement d'une représentation par une autre, mais plutôt concurrence, empilement, articulation et même complémentarité suivant des modalités variées qui dépendent des discours et des pratiques qu'elles fondent.

\subsection{L'offre de transport comme dispensatrice de richesse}

En ouvrant les marchés, en permettant la production et les échanges, le transport est une source de richesse en toutes circonstances et pour tous. Dans ce cadre, les effets de l'amélioration de l'offre de transport apparaissent de manière automatique. En ce sens, l'offre de transport constitue un actif économique- puisqu'elle produit des richesses - générique - puisqu'elle est à la disposition de tous. Voilà exposée brièvement la première représentation que l'on peut repérer concernant l'appréhension des liens entre transport et société.

Cette représentation est largement prégnante dans les discours politiques. Les débats récents qui ont agité le microcosme français autour de la nécessité de réaliser une ligne ferroviaire à grande vitesse entre Paris et Strasbourg en fournissent, après de multiples autres, un exemple édifiant. L'analyse du fonctionnement du discours politique et de son besoin de simplismes (ou de symboles) pourrait faire l'objet d'une application passionnante au champ des transports. Ce n'est pas l'objet du présent article. On notera simplement que, d'une part, l'alimentation du discours politique est un aspect important de la demande sociale s'adressant aux analystes des transports et, d'autre part, le discours politique véhicule une représentation déterministe en termes d'effets (habituellement positifs) de l'offre de transport sur la société.

Au niveau du discours politique, la remise en cause de cette représentation déterministe est aujourd'hui nettement perceptible. On peut en premier lieu se demander si elle ne participe pas d'un mouvement plus vaste de défiance des citoyens vis-à-vis du jeu politique. Mais c'est surtout de l'irruption des préoccupations écologiques dans les débats sur les infrastructures de transport que vient le changement. Les argumentaires construits sur une opposition au productivisme prennent par nature le contre-pied de ceux qui font de l'abaissement des obstacles aux déplacements le déterminant de progrès de nature socio-économique. Les oppositions, moins idéologiques, des riverains (le syndrome NIMBY - not in my backyard - dénoncé par les technocrates qui se posent en dépositaires de l'intérêt général) procèdent finalement de la même démarche lorsqu'elles reviennent à retenir des considérations de qualité de vie face aux prévisions de trafic des projeteurs. Les discours déterministes sur les effets socio-économiques de l'offre de transport ne sont donc plus les seuls tenus. Ils voient en outre leur efficacité, mesurée en terme de réalisations concrètes, fortement entamée.

Le même type de lien déterministe entre transport et société fonde en partie la méthode la plus utilisée en matière d'évaluation technico-économique des investissements dans le domaine des transports. Reposant sur quelques hypothèses de micro-économie classique, la méthode coûtavantage consiste en effet, comme son nom l'indique, à recenser puis à sommer les avantages et les inconvénients monétarisables qui peuvent être retirés d'un projet. Elle fonctionne donc complètement dans une logique linéaire puisqu'elle implique d'établir un lien de causalité simple entre un fait (un investissement) et ses conséquences socio-économiques (par exemple l'avantage que retirent les usagers des gains de temps qui leur sont procurés ou de la possibilité de se déplacer qui leur est offerte). Une critique de la méthode coût-avantage n'a pas sa place ici. On notera simplement avec intérêt que cette causalité simple n'apparaît plus aujourd'hui aussi opérationnelle 
que par le passé. La mesure du "trafic induit" par une infrastructure amène en particulier de plus en plus souvent les techniciens à envisager une relation plus complexe entre l'offre de transport et les usages (CEMT, à paraître)(1).

Enfin, au niveau de la théorie économique, les modèles les plus usuels, qu'ils traitent de localisation (WEBER, ISARD, MOSES), d'aires de marché (LÖSCH, HOTELLING) ou de l'usage du sol (VON THÜNEN), intègrent tous la distance à travers une fonction de coût de transport. Dans ce cadre, le lien est par nature direct entre l'amélioration de l'offre de transport et les conséquences généralement bénéfiques - que les entrepreneurs peuvent en retirer. De ce point de vue, le modèle core-periphery, proposé par Paul KRUGMAN et en vogue actuellement chez les économistes s'intéressant à l'espace, ne diffère pas de ses prédécesseurs. Les coûts de transport demeurent l'une des variables qui déterminent la localisation des activités (Calmette, 1994; Krugman, 1991). L'une de ses spécificités est cependant d'offrir plusieurs solutions pour une configuration donnée des variables. Ce sont alors les conditions initiales, l'histoire et ses aléas, qui vont influencer la solution finalement adoptée (2). Le modèle intègre donc une dose de contexte socio-historique et une pincée de rationalité des agents (par anticipation auto-prophétique) (Ragni, 1995). Il s’éloigne ainsi de la matrice linéaire initiale.

Les modèles spatiaux théoriques récents marquent de réels progrès. Les phénomènes qu'ils mettent en évidence permettent une compréhension nouvelle des évolutions passées. Cependant, les approches en terme de coûts de transport se révèlent foncièrement inadaptées à décrire un monde économique dans lequel la compétition ne porte pas seulement sur les coûts. Le développement d'une concurrence hors coûts, fondée en particulier sur la capacité d'innovation et de réaction, semble caractéristique des évolutions du système productif de l'après-fordisme (Veltz, 1993). Du point de vue des économistes aussi, l'approche déterministe paraît montrer ses limites.

Ce rapide examen montre en premier lieu que, concernant la relation transport-société, la représentation causale directe est encore largement actuelle au sens où elle fonde nombre de spéculations intellectuelles et de pratiques concrètes. Dans le même temps, il est apparu de plusieurs points de vue que les habits du déterminisme semblent aujourd'hui trop étroits pour continuer à avancer, sauf à accepter de les porter de manière moins rigide. Enfin, de multiples problématiques tentent désormais de se passer de ces oripeaux. C'est un bilan contradictoire qui se dégage finalement d'une représentation intellectuellement dépassée mais qui conserve une redoutable efficacité.

\subsection{L'offre de transport à valoriser par les acteurs}

La représentation développée ensuite a fait dépendre la valorisation d'une offre de transport de la capacité des acteurs à intégrer cette offre dans leur activité. On est là tout proche du discours qui fait du transport une condition permissive du développement. Selon cette vision, l'offre de transport n'est plus un actif - puisqu'elle nécessite une mise en valeur - mais une ressource. De la même manière, elle n'est plus générique, mais spécifique aux acteurs qui sauront la valoriser.

C'est dans ce cadre que se situe aujourd'hui l'essentiel du discours technico-économique concernant - la terminologie est éloquente - la valorisation des infrastructures de transport. Cette valorisation implique de la part des pouvoirs publics l'adoption de "mesures d'accompagnement" à même de concrétiser des potentialités. Elle conduit à des stratégies très volontaristes qui ne sont pas toujours couronnées de succès. A ce niveau, la rupture avec la période précédente est ténue puisque l'on discerne souvent, cachée sous les "mesures d'accompagnement", les mêmes prémisses fondamentalement déterministes. On a pu évoquer à ce sujet la "rhétorique des effets structurants conditionnels" (Claisse, Duchier, 1995).

Cette évolution légère des pratiques est liée à un renouvellement plus important au plan théorique. On assiste en effet, dans les milieux de la recherche appliquée aux transports et à

(1) Voir en particulier l'article de Phil B. Goodwin, "Trafic supplémentaire induit par la construction de routes : preuves empiriques, incidences économiques et implications politiques".

(2) Un autre modèle contemporain, développé par W. B. ARTHUR, adopte un point de vue identique (Arthur, 1995) 
l'aménagement, à la remise en cause de l'existence d'un lien de causalité simple entre l'amélioration d'une offre de transport et les transformations des structures économiques et sociales des zones desservies (Plassard, 1976). On affirme que le système de transport est produit par la société et qu'il participe, en retour, de son évolution. On désigne même les comportements de mobilité comme les principaux vecteurs de ce jeu d'actions et de rétroactions. Ces avancées importantes ont permis de proposer et de mettre en ouvre une méthodologie pour analyser l'insertion dans un contexte socioéconomique donné d'une modification de l'offre de transport qui repose justement sur l'observation des comportements de mobilité. Il y a donc lieu de distinguer les deux aspects, théorique d'une part et méthodologique de l'autre.

Au plan théorique, il faut bien avouer que l'effort n'a pas été poursuivi avec beaucoup d'assiduité dans ce domaine par les socio-économistes des transports. Ainsi, la nature des liens entre système de transport et système social n'a pas été systématiquement explorée malgré les ouvertures tentées autour de la notion de congruence (Offner, 1980). Le rôle essentiel dévolu à la mobilité n'a pas été suffisamment critiqué, sans doute parce qu'il fondait une méthodologie qu'il s'est ensuite agi de mettre en ouvre. Les voies par lesquelles la société produit l'offre de transport, en particulier, ont largement été délaissées.

Du point de vue des méthodes d'investigation, l'utilisation systématique d'enquêtes de mobilité a permis, d'un point de vue empirique, de progresser dans la connaissance des phénomènes qu'il s'agissait d'étudier. Le principal de ces progrès est venu de la prise en compte de variables caractérisant non plus seulement le coût de transport, mais également la nature des échanges réalisés. Le système explicatif mis en place à cette occasion s'est donc révélé davantage pertinent avec les évolutions du moment. L'analyse des évolutions des comportements de déplacements liés à la mise en service des TGV sud-est puis Atlantique a notamment permis de mettre en relation les transformations qualitatives du trafic à des dynamiques économiques ou sociologiques exogènes (Cointet-Pinel, Plassard, 1986; Klein, Claisse, 1997).

Néanmoins, l'effort de renouvellement n'a pas été assez conséquent pour éviter le piège de l'empirisme. S'appuyant sur les propositions de François PLASSARD, l'analyse s'est focalisée sur les évolutions concrètes de la mobilité. Dans les faits, seules des évolutions de court terme ont pu faire l'objet d'un examen rigoureux. En outre, la mise en oeuvre des observations s'est traduite par l'étude des comportements de déplacement plutôt que de la mobilité. D'une construction théorique initiale extrêmement large - le système de transport produit du système social - on est arrivé à des observations très ponctuelles. Non seulement celles-ci sont difficilement généralisables car spécifiques à un contexte géographique particulier et à une situation de l'offre de transport. Mais en plus, elles ne permettent pas de replacer les déplacements observés ni dans la sociabilité globale des individus enquêtés, ni dans l'économie générale des firmes pour lesquelles, le cas échéant, ils voyagent.

D'autres travaux, d'horizons divers, ont entrepris de contourner l'obstacle en faisant par exemple dépendre l'apparition d'éventuels effets découlant d'une modification de l'offre de transport des stratégies adoptées par les acteurs en présence. Mais les fondements de ces stratégies ne sont jamais totalement explicités. D’autres approches encore, modélisatrices, calculent de la même manière des " potentiels " plutôt que des prévisions (Burmeister, Joignaux, 1997). Ce faisant, dans l'un ou l'autre cas, la traduction en une réalité économique et sociale du fait technique de l'offre de transport est partiellement occultée. Ainsi, dans un cadre théorique pourtant construit avec cet objectif, les recherches menées depuis 20 ans sur le thème "transport et société" n'ont que très rarement permis d'explorer véritablement les interactions entre les deux termes.

\subsection{L'offre de transport partie prenante de la société}

Dans les recherches les plus récentes, l'offre de transport demeure en premier lieu le produit d'une société et d'un système technique qu'elle participe ensuite, à travers ses usages, à faire fonctionner, et donc à faire évoluer. Le renouvellement vient de la tentative de tirer de manière systématique les enseignements de cet axiome. Compte tenu de ce que les comportements de 
déplacements ne constituent vraisemblablement pas la seule voie d'interactions entre le système de transport et le système social, il convient alors de donner un contenu plus précis à ce processus d'échanges réciproques. Evidemment, plusieurs pistes ont été ouvertes. On peut mentionner, dans le champ de l'économie les approches théoriques autour de la notion de croissance endogène dans la mesure où elles réussissent à intégrer les dépenses publiques -notamment en transport- parmi les facteurs de croissance. D'autres analyses, s'appuyant sur les théories de la décision élaborées en sciences politiques, permettent de montrer que le processus décisionnel concernant les infrastructures est un processus social complexe.

Ces approches centrées sur les résultats d'une discipline, sont essentielles en ce qu'elles permettent, chacune sur leur champ, de réelles avancées de connaissance. La poursuite de ces recherches n'est donc pas antinomique de la quête d'une approche plus globale de la question des relations entre l'offre de transport et la société ; plus globale et donc par nature moins centrée sur une discipline académique unique. C'est dans cette optique que s'inscrit la démarche exposée cidessous.

A partir de ses travaux concernant la communication, Patrice FLICHY développe, dans un ouvrage récent, une théorie de l'innovation technique (Flichy, 1995). Elle repose en premier lieu sur l'affirmation selon laquelle la technique est une partie du social et qu'elle ne peut être envisagée séparément de celui-ci. L'innovation se développe donc au sein de "mondes sociaux" et du "cadre socio-technique" attaché à chacun d'eux. Les mondes sociaux se rencontrent autour "d'objetsfrontière" qui leur sont communs, lors de la combinaison de plusieurs innovations préalables dans une création plus complexe par exemple, ou encore lorsque les usagers se confrontent au nouvel objet qui leur est proposé et à ses concepteurs. Ces périodes voient aussi les cadres socio-techniques se combiner en un cadre plus large. Le second point que nous conserverons ici concerne les deux dimensions que l'auteur attribue aux cadres socio-techniques: ils sont à la fois "cadres de fonctionnement" liés à l'artefact technique et "cadres d'usage". On obtient alors l'image que nous conserverons de deux cadres -"de fonctionnement" et "d'usage" - immergés dans la société.

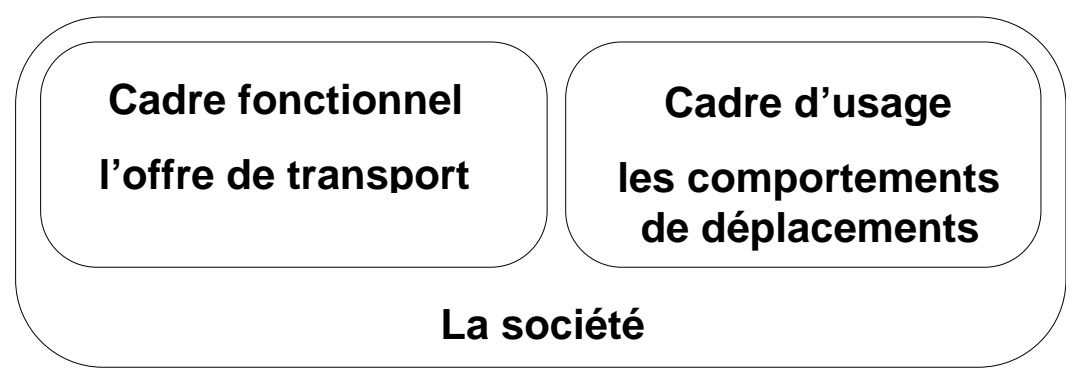

Figure 1 : Le cadre de l’innovation socio-technique

Pour utiliser cette construction afin d'appréhender la question des relations entre offre de transport et société, il convient seulement de donner un contenu plus précis au cadre fonctionnel et au cadre d'usage. Le cadre fonctionnel est dans une première approche largement assimilable à l'offre de transport, mais à condition de donner à ce dernier terme une large acception. Il englobe tout ce qui touche à l'artefact technique. Bien qu'ils aient leur place dans cette grille de lecture, nous n'aborderons pas ici les aspects concernant la technologie ferroviaire à grande vitesse. Nombre de travaux sont d'ores et déjà mobilisables pour donner corps de ce point de vue au cadre socio-technique de son développement (Revue d'Histoire des Chemins de Fer, 1995). Nous nous en tiendrons à qualifier les services mis à la disposition des usagers. Au sein de notre problématique, le cadre d'usage renvoie à la mobilité, à une conception élargie de la demande de transport. L'objectif est ici avant tout de réexaminer, à travers une grille de lecture rénovée, les résultats d'enquêtes de mobilité réalisées autour des TGV sud-est et Atlantique. Un niveau transversal, dénommé « la société » dans le schéma précédent, est composé d'éléments formant le contexte spatial, temporel et idéologique des deux cadres identifiés plus haut.

La représentation proposée par Patrice FLICHY doit beaucoup à la sociologie interactionniste. A ce titre, elle implique d'articuler des considérations micro- et macro-sociales. C'est en particulier 
à cette articulation que renvoie la notion de "monde social". Il s'agit en fait de distinguer différentes échelles de phénomènes. Tout n'est pas explicable au niveau local, ou à court terme. Tout n'est pas relatif à l'individu. En revanche, toutes les évolutions ne renvoient pas au destin de la planète ou à celui du capitalisme. Il convient donc d'intégrer la réalité de ces différents niveaux et de confronter les transformations qui y trouvent place.

Selon ces prescriptions, nous distinguerons de manière schématique deux niveaux : un niveau global (partie 2), où seront présentées à grands traits les principales évolutions pouvant être inscrites dans notre champ des déplacements interurbains à motif professionnel effectués à grande vitesse, et un niveau local (partie 3), où la situation particulière de différentes agglomérations sera détaillée. A l'intérieur de chaque niveau, nous préciserons d'abord l'environnement social avant d'aborder le contenu des cadres de fonctionnement et d'usage définis plus haut.

\section{CADRE DE FONCTIONNEMENT ET CADRE D'USAGE : DES TENDANCES LOURDES}

L'approche globale renvoie nécessairement à des évolutions de société de grande portée. On comprendra dès lors qu'il n'est pas question en quelques pages de détailler des tendances qui font par ailleurs l'objet de multiples analyses. On se résoudra donc à ne trouver ici qu'un minimum d'explication sur ces trends sociaux. Le plan de cette présentation est imposé par la grille d'analyse adoptée : quelques éléments du contexte socio-économique général (2.1) avant de donner un contenu au cadre fonctionnel (2.2) puis au cadre d'usage (2.3) des déplacements à grande vitesse.

\subsection{L'environnement socio-économique}

Nous retiendrons deux faits qui nous semblent caractéristiques des évolutions de la société contemporaine : la forte valorisation sociale de la vitesse et de la circulation (2.1.1) d'une part, la mutation du système productif (2.1.2) d'autre part. Ces deux événements ne s'inscrivent pas dans notre époque selon les mêmes rythmes ni les mêmes modalités. La circulation est une valeur fondatrice du capitalisme que vantaient déjà les économistes du XVIIIème siècle. Elle n'a depuis cessé de s'imposer à l'économie, la politique, l'urbanisme, les modes de vie et a acquis une indéniable dimension culturelle. Elle s'inscrit sur le long terme. La mutation actuelle du système productif paraît en regard tout à fait conjoncturelle. Elle démarre au mieux à la fin des années 60 avec l'ébranlement progressif des fondements de l'organisation fordiste et du mode de régulation qui lui est attaché. Elle n'a pas encore conduit à une stabilisation autour d'un modèle paradigmatique aussi achevé que celui qu'elle met à bas.

\subsubsection{Circulation}

Il est parfaitement clair que le commerce est à la base du développement du capitalisme. Le marché est alors le lieu de la circulation des biens. Pierre ROSANVALLON montre bien comment dans l"'utopie libérale" qui émerge au XVIIIème siècle, le concept de marché évolue ; il ne désigne plus, pour Adam SMITH, "un lieu particulier et localisé d'échange : c'est la société toute entière qui le constitue" (Rosanvallon, 1989). Il est devenu le mode de régulation sociale d'une idéologie opposée à la notion de contrat de Jean-Jacque RousSEAu.

Il ne faut certes pas confondre cette idéologie circulatoire utopique avec la pratique économique de la bourgeoisie "en situation de gestion de la société". Il n'en demeure pas moins que l'idée de marché et la fluidité qui l'accompagne fonde en partie notre organisation sociale au-delà même de l'économie.

Dans ces conditions, on peut s'interroger sur le statut d'une autre utopie, plus récente : l'utopie communicationnelle. Celle-ci est apparue à l'occasion des recherches qui allaient fonder la cybernétique et a très rapidement acquis une dimension idéologique, notamment sous l'impulsion de Norbert WiEnER. Il s'agissait d'"interpréter le réel tout entier en terme d'information et de communication", et de "reconnaître la communication comme valeur centrale pour l'homme et la société". Sur cette base, Philippe BRETON analyse une idéologie représentant un homme nouveau qui tire son essence de la communication et non de ses qualités propres. Parallèlement, "la nouvelle 
société s'articule autour du thème de la transparence sociale" et se construit, non pas par exclusion de certains de ses membres, mais au contraire à travers l'intégration de tous (Breton, 1992). Cette dimension sociale met en lumière le fait que l'utopie communicationnelle est aussi née d'une réaction à la barbarie que portent en elles les trois idéologies héritées du XIXème siècle, fasciste, communiste et libérale.

Il faut pourtant reconnaître que la rupture avec le capitalisme n'est pas totale. La "pratique communicationnelle", non conflictuelle par nature, est essentiellement individualiste. En ce sens, elle s'insère dans notre société sans (encore ?) la remettre en cause. Elle se nourrit de ses productions qui sont justement les activités aujourd'hui dominantes (informatique, télécom...) alors qu'en retour, le marketing de ces industries abuse des image de l'utopie communicationnelle. Les deux idéologies se retrouvent enfin sur l'exigence de fluidité. La valorisation économique et sociale de la circulation s'appuie sur une double valorisation idéologique.

\subsubsection{Mutations du système productif}

La crise du modèle fordiste d'organisation productive est aujourd'hui patente. Ni le mode de régulation macro-économique qu'il impliquait, ni les techniques productives qu'il mettait en œuvre et ni les liens sociaux qui le fondaient ne sont épargnés par cette crise multiforme (3). Nous n'en retiendrons ici que l'importance toute particulière prise par la notion de flexibilité qui, considérée d'un point de vue quantitatif ou qualitatif, conjuguée sur le court ou le long terme, au niveau stratégique ou opérationnel, etc., est l'une des clés d'adaptation à une situation de concurrence intensifiée. Le système productif est donc en mutation profonde. Bien qu'il ne semble pas devoir se stabiliser à un horizon prévisible, on peut néanmoins distinguer deux types d'évolutions différents.

Nous partirons d'une remarque de Pierre VeLTZ selon lequel la concurrence entre les firmes s'articule autour de deux dimensions : la compétition par les prix et la compétition "hors-coût", reposant sur la qualité, l'adaptation à la demande, l'innovation, etc. (Veltz, 1993). L'importance croissante de ce deuxième aspect alors que la pression sur les prix est toujours très intense est caractéristique des mutations actuelles du système productif. Pour répondre à ce double enjeu, l'organisation des entreprises s'est trouvée profondément transformée. Schématiquement, on est passé d'une structure pyramidale et hiérarchique à une structure en réseau, ou cellulaire. Mais cette autonomie des "cellules" de production va de paire avec un renforcement du cadre général de l'organisation et la soumission à ses objectifs. On a donc un double mouvement d'intégration organisationnelle et d'autonomie des structures.

Cette évolution se repère à tous les niveaux du système productif : à l'intérieur des firmes depuis la répartition des compétences entre filiale jusqu'à la gestion du travail individuel au sein d'une équipe. On est passé d'un contrôle a posteriori des actes à un contrôle a priori des structures et des hommes qui permet de leur laisser davantage d'autonomie.

Pour avérée que soit cette tendance, elle ne fait pas l'unanimité chez les analystes. Les sociologues du travail en particulier notent la persistance, voire le renforcement de pratiques tayloriennes (Sociologie du travail, 1993). Dans cette optique, la recherche de flexibilité se traduit par l'emploi précaire renforcé, par une déqualification des fonctions, etc. Cette seconde direction paraît tout aussi présente que la première. Elle serait même quantitativement dominante. Nous empruntons à Jean-Pierre DuRAND l'expression de "taylorisme flexible" pour la dénommer (Durand, 1991).

(3) Concernant l'analyse de la crise du fordisme, on pourra se reporter notamment à (Boyer, 1992; Mazier, Basle et Vidal, 1993). 


\section{Compétition par les coûts}

\section{Compétition hors-coûts}

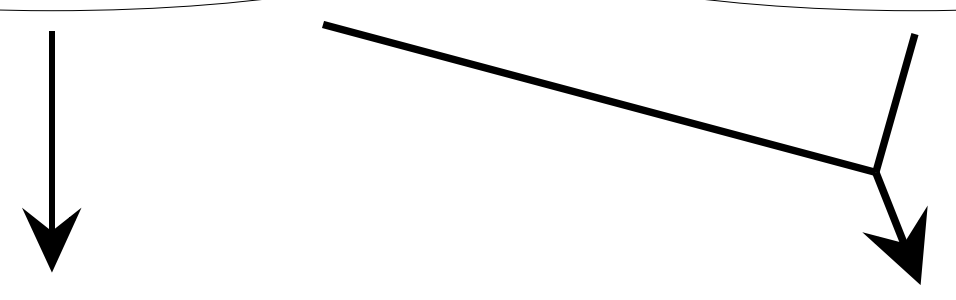

\section{Taylorisme flexible}

\section{Intégration / Autonomie}

\section{Figure 2 : les deux évolutions du système productif}

Loin d'être exclusives, les deux évolutions coexistent au sein d'un système productif dualisé. Le double mouvement d'intégration-autonomie serait caractéristique des situations où la concurrence se joue sur les deux aspects, sur les prix et hors les coûts. Le taylorisme flexible s'imposerait lorsque la pression sur les prix est le principal déterminant du jeu de l'offre et de la demande.

\subsection{Le cadre fonctionnel}

Au niveau global qui est le nôtre, nous retiendrons, pour donner un contenu au cadre fonctionnel de l'offre de transport à grande vitesse, trois phénomènes distincts. Le premier concerne, sur une longue période, l'évolution continue des coûts - à la baisse - et des vitesses - à la hausse - de déplacement (2.2.1). Le second se limite au constat selon lequel les coûts sociaux des transports ne sont pas encore un obstacle à la mobilité interurbaine en Europe (2.2.2). Le troisième tient à la logique particulière de développement de l'offre de transport à grande vitesse (2.2.3).

\subsubsection{Un mouvement continu d'accélération et de baisse des coûts}

Mieux qu'un long discours, le graphique suivant illustre la diminution des temps de parcours en France sur deux siècles. Il est fondé sur la mesure de temps de parcours terrestres (poste et diligence puis chemin de fer) au départ de Paris (4). Le dernier point, correspondant à l'année 2010, a été estimé en faisant l'hypothèse, dont on sait qu'elle est erronée, d'une réalisation intégrale du schéma directeur des lignes ferroviaires à grande vitesse adopté en France.

On constate alors que l'accélération des déplacements est un mouvement continu qui a une origine antérieure à l'apparition du chemin de fer. On vérifie ensuite que la construction du réseau au XIXème siècle s'est traduit par une amplification du phénomène jusqu'en 1880. Ensuite, l'amélioration des infrastructures existantes a encore entretenu cette tendance tout au long du XXème siècle. Enfin, le dernier point, même bâtit sur une hypothèse exagérément optimiste, démontre que le TGV ne fait que s'inscrire dans un mouvement séculaire.

(4) Les données antérieures à 1940 ont été rassemblées par Christophe STUDENY (STUDENY, 1990). Celles concernant la situation 1980 ont été relevées sur les indicateurs SNCF. 


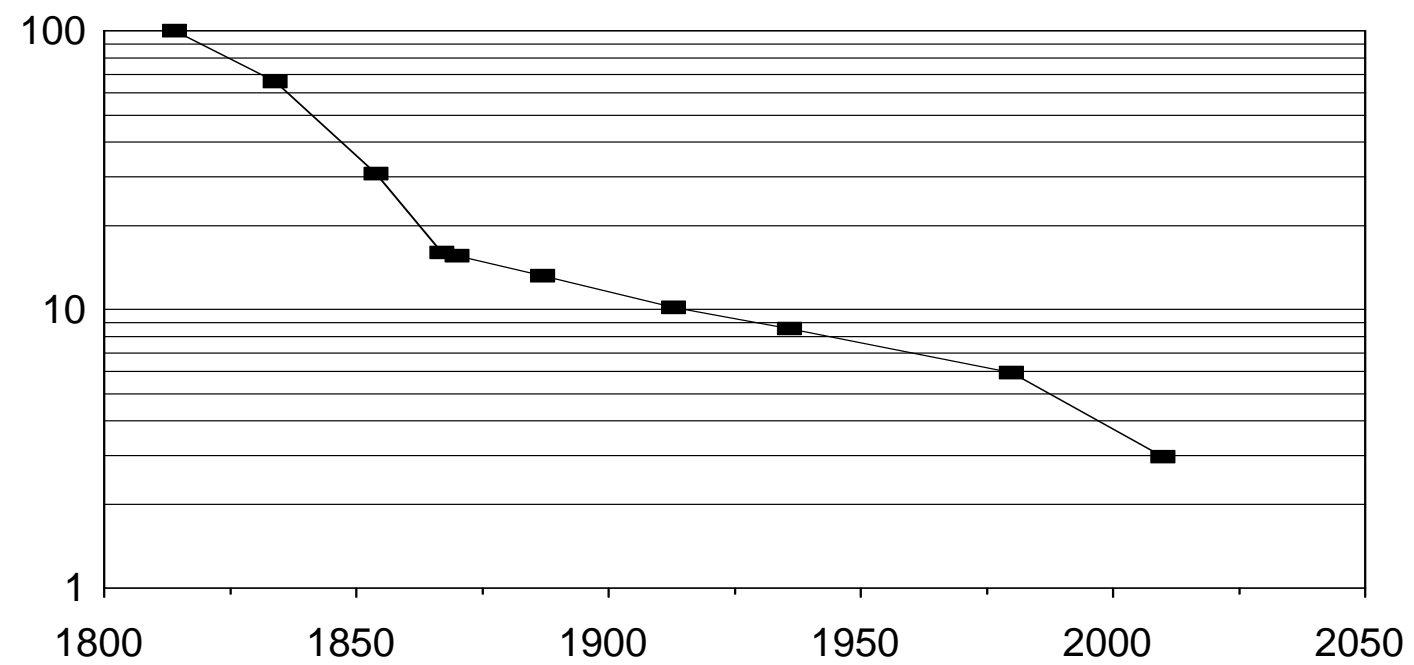

\section{Figure 3 : Evolution des temps de parcours terrestres au départ de Paris, 1814-2010}

Concernant la composante strictement monétaire des coûts de déplacements, la tendance est évidemment similaire. Elle est seulement moins facile à mettre en évidence de manière quantitative en raison des multiples précautions qu'implique la comparaison de prix pratiqués à des époques différentes. Il faut en premier lieu pouvoir ramener à un référent unique des unités de compte différentes. Il convient également, si l'on souhaite évaluer l'incidence du coût monétaire comme obstacle au déplacement, introduire dans l'analyse l'évolution des niveaux de vie. Enfin, il est nécessaire de déterminer précisément les niveaux de service (vitesse, confort, etc.) que l'on va comparer à chaque étape.

Devant ces difficultés, il reste à accumuler des présomptions. Sur le long terme, on se référera à Jean-Claude TouTAIN qui met en évidence une décroissance continue des tarifs ferroviaires de 7,1c/voyageur.kilomètre en 1840 à 4c/V.K en 1913 (Toutain, 1967). Concernant la période contemporaine, la baisse des coûts du transport aériens est un fait avéré depuis 30 ans au moins. Depuis le début des années 80, l'avion et le TGV sont en concurrence sur un nombre de relations croissant à mesure de l'extension du réseau de lignes ferroviaires nouvelles. Une fois la desserte TGV mise en place, les performances techniques des deux moyens de transport sont à peu près figées. La compétition se déroule alors largement sur les aspects tarifaires indiquant par là que le TGV s'inscrit lui aussi dans un processus séculaire de diminution des prix du transport.

\subsubsection{Les coûts sociaux encore ignorés}

Les coûts sociaux des déplacements à longues distances ne viennent pas encore réduire de manière significative la mobilité. En France, cette réalité est confirmée concernant la mobilité urbaine par tous les analystes (Orfeuil, 1994). Les déplacements interurbains sont en comparaison encore moins sensibles à la congestion ou aux atteintes à l'environnement.

Il n'est certes pas impossible que la situation évolue dans ce domaine à brève échéance. Les incitations à maîtriser la mobilité sont fortes. D'autres pays semblent davantage engagés dans cette voie. Mais il convient de souligner que partout, les inerties sont fortes. Dans les situations les plus favorables, les efforts pour internaliser (faire prendre en charge par l'usager) les coûts de pollution n'en sont qu'aux balbutiements. Mais c'est surtout au niveau des modes de vie, de l'urbanisation et des modes d'organisation des entreprises que les rigidités sont importantes. Aujourd'hui, le développement des loisirs, la péri-urbanisation ou l'adoption de logiques d'organisation circulatoires, pour ne citer que quelques tendances consommatrices de déplacements, ne semblent remises en cause que de manière marginale. On s'en tiendra donc à cette image de coûts sociaux encore largement ignorés. 


\subsubsection{La logique de la grande vitesse}

L'accélération des déplacements s'accompagne d'une mutation de l'offre de transport qui dépasse le simple aspect des temps de parcours. La morphologie même des réseaux d'infrastructures évolue en effet dans le sens d'une massification des flux et d'une raréfaction des points d'entrée. Les gares, et plus encore les nœuds, du réseau TGV acquièrent donc une position tout à fait particulière qui les distinguent fortement du territoire qui les entoure.

Elles bénéficient en effet de l'addition de deux avantages comparatifs : l'un concernant la vitesse, l'autre concernant la disponibilité de l'offre. A l'aide d'une simulation théorique consistant à superposer un réseau rapide sur un espace banal, on peut illustrer de manière simple les conséquences en terme d'accessibilité de cette logique de la grande vitesse (d'après (Plassard, 1989)). Le point central du réseau bénéficie à plein d'une accessibilité renforcée à un large espace symbolisée ici par les couleurs claires. Si l'on choisit en revanche un point non connecté, l'espace s'assombrit. On retrouve en outre une structure de proximité concentrique alors qu'elle était très déformée par le dessin du réseau dans le premier cas. On se déplace dans cet exemple 4 fois plus vite sur le réseau rapide que sur l'espace banal.

Ces évolutions de la morphologie des réseaux résultent en première approche du mouvement d'accélération des flux. Des analyses plus fines montrent qu'elles dépendent également d'un ensemble d'options qui renvoient explicitement à l'élaboration du cadre fonctionnel. On peut illustrer ce propos en précisant ce qui différencie les conceptions française et allemande de la grande vitesse ; partant d'un objet technique très voisin, les premiers ont mis en place un ensemble de dessertes à longue distance concurrentes de l'avion, très centralisé autour de Paris, avec une complémentarité des réseaux classique et nouveau limitée à la prolongation des parcours des TGV ; les seconds ont développé un système de desserte intégrant totalement grande vitesse et trains classique, irrigant l'ensemble du territoire, mais très orienté sur les distances intermédiaires. On distingue facilement derrière ces caractéristiques de l'offre, les spécificités de chaque pays qui ont pu participer à structurer le réseau de telle ou telle manière.

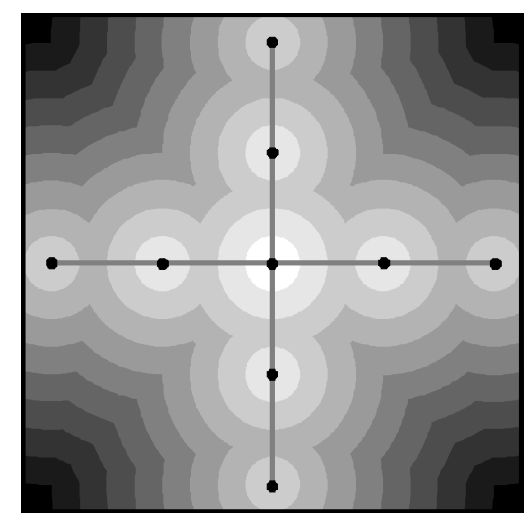

Au départ du noeud central du réseau

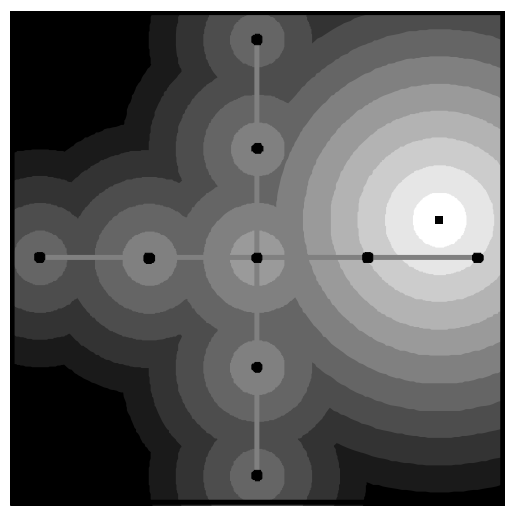

Au départ d'un lieu isolé du réseau

\section{Figure 4 : Accessibilité et réseau rapide : une approche théorique}

\subsection{Le cadre d'usage}

D'un certain point de vue, on aurait pu inscrire dans le cadre d'usage de l'offre de transport à grande vitesse les évolutions présentées dans le contexte socio-économique. Il est par exemple clair que la forte valorisation sociale de la circulation trouve un écho particulier dans le développement 
toujours plus intense de l'usage des modes de déplacement les plus rapides. De même, l'existence d'un lien entre les nouvelles formes de l'organisation productive et les besoins de déplacements est évidente. Il restait, à travers la description du contexte socio-économique, à rendre compte d'une dimension des phénomènes mentionnés beaucoup plus large que leur implication directe en termes de transport.

Dans cette optique, le cadre d'usage trouve son contenu dans la pratique sociale qui se développe autour de l'offre de transport à grande vitesse. Nous en traiterons deux aspects. Le premier concerne la permanence d'une forte différenciation sociale à travers l'usage des moyens de déplacement les plus rapides (2.3.1). Le second décrit deux modes d'usage de la grande vitesse : comme opportunité pour réduire le coût du déplacement ou comme nécessité pour adopter de nouveau modes de vie ou d'organisation (2.3.2).

\subsubsection{Vitesse et différenciation sociale}

A chaque époque, les moyens de transport les plus rapides ont été mis en ouvre par les structures sociales et les activités dominantes. Cette réalité se décline d'abord par rapport à la hiérarchie des villes. Concernant le développement du chemin de fer au XIXème siècle, Denise PuMAIN affirme ainsi que "[...] le réseau a surtout été dessiné en fonction d'une hiérarchie de taille et de dynamismes urbains qui préexistaient à sont installation" (Pumain, 1982). Cette priorité donnée aux liaisons entre les grandes concentrations urbaines demeure de nos jours. En France, la première grande liaison autoroutière achevée reliait Lille, Paris, Lyon et Marseille, la première ligne TGV, Paris et Lyon.

Mais la concordance des performances élevées des moyens de transport et des sommets de la société se repère aussi sur d'autres dimensions. Ainsi, les activités qui concentrent pouvoir et capacité à produire de la valeur ajoutée sont-elles toujours les premières utilisatrices des moyens de déplacements rapides. Fernand BRAUDEL a abondamment illustré ce phénomène concernant la diffusion des nouvelles, des lettres de changes ou des épices (Braudel, 1979). Aujourd'hui la réalité est évidemment moins tranchée. On remarque néanmoins la propension particulière des activités de direction et de gestion d'une part, de celle liées à la conception des produits, à l'innovation ou à l'articulation production/marché d'autre part à consommer du déplacement à grande vitesse. Il n'est pas anodin de retrouver ce couple pouvoir-richesse (5).

Enfin, on retrouve cette concordance de manière presque caricaturale si l'on s'intéresse à la position sociale des usagers des modes de transports les plus performants. Sous l'ancien régime, le cheval est l'apanage de la noblesse. A notre époque, toutes les observations montrent que l'accès et l'usage des moyens de déplacement rapides sont en premier lieu le fait des individus au statut socioprofessionnel privilégié. L'enquête de mobilité réalisée autour du TGV-Atlantique montre par exemple une sur-représentation des catégories aisées parmi les voyageurs quel que soit le mode considéré. Mais à ce phénomène statique de différenciation sociale s'ajoute une dimension dynamique. On observe en effet que la majeure partie du trafic supplémentaire capté par le TGV ressortit de ces mêmes catégories aisées (Klein, Claisse, 1997). A la revalorisation technique d'une offre de transport répond donc une revalorisation sociale de sa clientèle.

Quel que soit le point d'entrée adopté, la conjonction des structures sociales dominantes et de la performance des moyens de déplacement apparaît comme une constante du cadre d'usage que nous cherchons à décrire.

\subsubsection{La grande vitesse : opportunité ou nécessité}

Le second aspect constitutif du cadre d'usage que nous souhaitons exposer ici tient davantage, il faut bien le reconnaître, de l'hypothèse de travail que de l'analyse partagée par les observateurs de la mobilité. Il s'agit de considérer que la traduction sociale - à travers les usages - des performances

(5) On notera également avec intérêt que les activités liées aux moyens de communications les plus rapides sont à chaque époque dominantes : le chemin de fer puis l'automobile et enfin l'informatique et les télécommunications. 
techniques d'un moyen de déplacement à grande vitesse s'opère par deux mécanismes distincts. Suivant le premier, la grande vitesse est une opportunité de réaliser plus rapidement une activité qui, même sans amélioration de l'offre de transport, devra se dérouler. Des gains de temps sont ainsi offerts dans le cadre d'un mode d'organisation ou d'un mode de vie qui ne sera pas modifié. L'opportunité sera saisie en fonction d'un arbitrage qui peut être du type coût-avantage.

Le second mécanisme met en œuvre une logique de nécessité. Il décrit une situation dans laquelle les gains de temps offerts sont une condition sine qua non de la réalisation de l'activité envisagée. La grande vitesse est alors une nécessité au regard du mode d'organisation ou du mode de vie adopté.

Deux exemples caricaturaux mais concrets peuvent peut-être aider à se faire une idée de ces deux mécanismes. Lorsqu'un cadre voyageant régulièrement pour affaires tout d'abord, va adopter le TGV plutôt que l'avion parce que cela lui permet de gagner du temps sur son trajet de porte à porte et de dépenser moins, il inscrit son usage du TGV dans une logique d’opportunité. En revanche, une entreprise pourra profiter de cette nouvelle facilité de déplacement entre deux sites pour mieux répartir l'activité entre eux et les spécialiser afin de réaliser quelques économies d'échelle. Complémentaires dans un cycle de production, les deux implantations devront communiquer davantage. Nous somme là dans une logique où la grande vitesse devient une nécessité au regard du mode d'organisation adopté. Evidemment, la réalité est toujours composée des deux aspects.

L'intérêt de cette construction théorique est double. D’une part, elle permet d'esquisser un lien entre le cadre d'usage et le cadre fonctionnel. Patrice FLICHY distingue en effet deux types d'usagers : l'usager-tacticiens, qui chercher à utiliser à son profit l'objet technique qui lui est proposé, et l'usager-stratège dont l'action va tendre à le modifier. La logique d'opportunité se rapporte assez bien au premier de ces comportements. La relation entre la logique de nécessité et l'usager-stratège est plus ténue mais demande à être précisée autour du couple évolution des modes de vie/évolution des techniques.

Cette construction théorique relie d'autre part l'usage des modes de transport à des dimensions plus générales de l'organisation sociale. Elle permet par exemple, tout en intégrant la dimension technique de l'offre de transport, de renvoyer l'usage de la grande vitesse comme opportunité au fonctionnement du système productif suivant le "taylorisme flexible". Au contraire, la grande vitesse comme nécessité rappelle aisément une structuration par "autonomie-intégration" (Klein, 1995).

\section{Une liaison TGV AVEc PARIS : DES Situations DifFERENCIEES}

Abandonnant les considérations générales, il s'agit maintenant de se rapprocher des situations concrètes dans lesquelles se déroule la mobilité interurbaine à motif professionnel de manière à préciser comment l'offre de transport à grande vitesse s'y inscrit. Nous disposons pour ce faire d'un riche matériau constitué par deux enquêtes lourdes destinées à relever l'évolution des pratiques et des motifs de déplacements avant et après la mise en service des TGV sud-est et Atlantique. La présentation de ces investigations et de leurs résultats ont fait l'objet de publications du Laboratoire d'Economie des Transports (Bonnafous, Patier-Marque et Plassard, 1981; Klein, Claisse, 1997; Plassard, Routhier, 1986). Nous y renvoyons le lecteur. Il faut néanmoins indiquer que l'enquête sud-est s'est déroulée en 1980 et 1985 et qu'elle permet surtout d'observer les relations entre Paris et Lyon. Ce sera notre premier exemple. L'enquête Atlantique est intervenue en 1989 et 1993 et concernait une aire géographique plus étendue. Nous présenterons ici les cas des relations de Tours, Nantes et Bordeaux avec Paris.

Suivant le plan imposé par la grille de lecture que nous avons adoptée, nous détaillerons en premier lieu le contexte socio-économique dans lequel s'inscrivait chacune des relations considérées lorsqu'elle a été observée (3.1). La description du cadre fonctionnel consistera à préciser les caractéristiques de l'offre de transport propre à chaque cas ainsi que son évolution (3.2). Le cadre d'usage sera déterminé par l'analyse des comportements de mobilité (3.3). 


\subsection{Diversité temporelle, diversité géographique}

Les quatre villes retenues pour leurs relations avec Paris présentent des caractéristiques très différentes, mais, nous voudrions en premier lieu nous attacher à la dimension temporelle qui vient distinguer les quatre exemples choisis. Il s'agit d'insister sur le fait que l'on observe les interactions entre l'offre de transport à grande vitesse et la société, non seulement en un lieu géographique déterminé par l'aire de chalandise des gares desservies, mais aussi à une époque conditionnée elle par les dates d'observations. A ce titre, l'analyse simultanée des enquêtes sud-est et Atlantique est particulièrement révélatrice de cette dimension temporelle du phénomène.

\subsubsection{La conjoncture : un élément-clé de l'analyse}

Les observations réalisées entre Paris et Lyon permettent de comparer la mobilité d'affaires entre les deux agglomérations en 1980 (sans TGV) et en 1985 (avec TGV). Les différences que l'on constate peuvent être reliées à la modification de l'offre de transport intervenue entre ces deux dates, mais aussi à l'évolution de la conjoncture, et plus largement à toutes les transformations de nature socio-économiques qui ont pu survenir dans l'intervalle.

1980 et 1985 présentent peu de différences du point de vue du taux de croissance, respectivement $+1,6$ et $+1,9 \%$. La dynamique des deux périodes est cependant très différente puisque la croissance a fortement diminué au début des années 80 pour se redresser, justement à partir de 1985. Au sein de cette tendance globale, quelques évolutions structurelles sont apparues qui donneront leur plein effet lors de la reprise marquée qui a suivi (1986-1990). Retenons d'abord la croissance spécifique de l'immobilier d'affaire qui permettra d'accompagner l'aménagement du quartier de la gare Part-Dieu à Lyon, mais aussi le développement particulier des activités de service aux entreprises. Enfin, on peut faire l'hypothèse que cette période d'intense restructuration a vu les entreprises rationaliser leurs différentes implantations sur le territoire national, la période suivante étant davantage marquée par un effort de redéploiement au sein de l'union européenne.

Entre 1989 et 1993, les évolutions sont très différentes. La conjoncture générale tout d'abord, s'est très fortement dégradée, 1993 étant une année de récession alors que 1989 a plutôt été le sommet de la vague de croissance. La crise particulière de l'immobilier d'affaire a fortement réduit les opérations d'urbanisme couplées avec l'arrivée du TGV. Les constructions de surfaces de bureaux ont été particulièrement affectées. Très sensible à la conjoncture, le secteur des services, et notamment celui destiné aux entreprises a connu une période très noire. Plus que lors de l'enquête sud-est, la situation économique générale a fortement influé sur les résultats de l'enquête Atlantique. Mais cette influence ne traduit que l'effet de la crise sur la mobilité. Il n'y a donc pas lieu d'observer l'insertion du TGV dans son contexte en excluant les périodes de crise, sauf à imaginer, en dépit de la réalité, un avenir sans récession économique.

Enfin, il convient de mentionner ici que 1993 restera aussi comme l'année d'une crise spécifique au chemin de fer. Les "ratés" de la mise en service du système de commercialisation "socrate" étaient pour l'essentiel résolus en septembre lorsque ont été recueillies les données de l'enquête. Ni les dysfonctionnements des mois précédents, ni l'image du train, et en particulier du TGV, onéreux n'étaient pour autant oubliés. Cette année-là, la SNCF a perdu $7 \%$ de son trafic grande ligne.

\subsubsection{Les caractéristiques de quatre agglomérations françaises}

Lyon, Tours, Nantes et Bordeaux se distinguent d'abord par la taille de leur agglomération : 1,2 M pour Lyon, 280.000 pour Tours, 500.000 à Nantes et 700.000 à Bordeaux. Elles diffèrent également par l'aire sur laquelle elles rayonnent, par le type de fonctions qu'elles assurent et par la richesse des activités de leur tissu économique. Pour une analyse détaillée de la place occupée par ces quatre cités, on se reportera utilement à l'ouvrage "La France en ville" publié par la DATAR (Damette, 1994).

A ce titre, Lyon apparaît comme un cas particulier. Elle est, Paris mise à part, la seule dont le caractère métropolitain semble indiscutablement dépasser le cadre de l'hexagone. D'une part, elle 
joue le rôle de capitale de la seconde région de France. D'autre part, elle exerce ses activités au sein de réseaux de relations qui dépassent largement cet espace. Très loin derrière Paris, elle réussit néanmoins à conserver, dans le domaine économique, des activités de coordination et de régulation non négligeables. Le tissu économique lyonnais est très diversifié et paraît bénéficier d'un certain degré d'autonomie sur des activités stratégiques. Dans un contexte général qui a pu évoluer depuis, ces caractéristiques étaient pour l'essentiel déjà valides au début des années 80.

Du point de vue de leur rayonnement, Nantes et Bordeaux présentent des profils assez proches. Parmi les premières agglomérations françaises, elles assurent un rôle de capitale régionale sur une aire étendue, dépassant les limites administratives. Il semble en revanche qu'elles ne pèsent pas d'un poids suffisant pour développer de manière significative des fonctions d'intermédiation d'envergure supérieure. Elles peuvent, à l'instar de Lyon mais à un degré moindre, s'appuyer sur un tissu économique diversifié. Elles semblent toutefois moins autonomes sur un certain nombre de "fonctions supérieures" analysées par Félix DAMETTE. Nantes et Bordeaux apparaissent typiques des métropoles françaises de province.

L'aire d'influence de Tours est indubitablement plus réduite, limitée par la concurrence des ses voisines, Nantes, Poitiers et Orléans. Elle se situe surtout dans l'aire d'influence de proximité de Paris. Enfin, l'activité de Tours apparaît moins orientée vers le secteur économique et davantage vers ce que Félix DAMETTE dénomme la "reproduction élargie" (santé, enseignement supérieur, etc.). Dans ce contexte, Tours peut néanmoins se prévaloir d'une diversité importante, d'une base commerciale active et de relations avec Paris plutôt dynamiques.

\subsection{L'offre de transport : un TGV peut en cacher un autre}

L'offre de transport sur une relation doit être appréciée de deux manières complémentaires : de manière absolue tout d'abord, en s'intéressant à ses dimensions quantifiables telles que le temps de parcours, la fréquence ou le prix (3.2.1), relativement aux opportunités d'usage qu'elle ouvre ensuite, afin de préciser le domaine des possibles (3.2.2). Mais il s'agit aussi d'observer ici une situation dans laquelle les performances du chemin de fer ont considérablement évolué. Il est donc également nécessaire de détailler les positions respectives de chacun des modes en présence.

\subsubsection{Les aspects quantifiables de l'offre}

Pour la description physique de l'offre, nous n'avons retenu dans le tableau suivant que les ordres de grandeur relatifs aux temps de parcours. La fréquence de desserte ferroviaire est en effet peu discriminante sur les quatre agglomérations étudiées qui bénéficient toutes de cadences élevées (depuis 14 services quotidiens entre Paris et Tours jusqu'à plus de 20 entre Paris et Lyon).

La réduction de temps de parcours est donc maximale sur Paris-Lyon, tant en valeur absolue qu'en valeur relative. Sur les relations du TGV-A, le pourcentage de temps gagné diminue régulièrement avec la distance. On retrouve la même hiérarchie à propos de la vitesse commerciale. Concernant les modes concurrents, on admettra que sur les trois relations desservies, les temps de parcours calculés d'aéroport à aéroport sont équivalents et ne peuvent pas être estimés à moins de $1 \mathrm{~h} 30$ compte tenu des délais de précaution et d'embarquement propres au transport aérien. On note enfin des différences de kilométrage significatives pour les parcours autoroutiers.

\section{Tableau 1 : l'offre de transport}

\begin{tabular}{lllll} 
de Paris à... & $\begin{array}{l}\text { train classique } \\
\text { (situation avant) }\end{array}$ & TGV & Avion & Autoroute \\
\hline Lyon & 4 heures & 2 heures & Oui & $450 \mathrm{~km}$ \\
Tours & 1h45 & 1 heure & Non & $230 \mathrm{~km}$ \\
Nantes & 3 heures & 2 heures & Oui & $380 \mathrm{~km}$ \\
Bordeaux & 4 heures & 3 heures & Oui & $580 \mathrm{~km}$ \\
\hline
\end{tabular}

On se limitera, concernant les aspects tarifaires, à souligner deux éléments de différentiation importants. La mise en service du TGV entre Paris et Lyon tout d'abord, a été réalisée à tarif 
constant (ou presque puisque qu'au tarif en vigueur sur le train classique s'est ajouté le montant de la réservation, soit 14F en 1985). L'introduction du TGV-Atlantique s'est au contraire accompagné d'une hausse conséquente du prix du trajet à travers la réservation devenue beaucoup plus onéreuse. Le second élément tient justement à ce que le montant de cette surtaxe est forfaitaire. Il pénalise donc davantage les trajets les plus courts. On peut ainsi calculer que le temps gagné par le TGV est, en 1993, vendu à l'usager près de $25 \%$ plus cher sur Paris-Tours que sur Paris-Nantes. Quoi qu'il en soit, dans tous les cas où l'avion est en concurrence avec le train, l'introduction du TGV a sauvegardé un différentiel tarifaire globalement favorable au fer.

\subsubsection{Franchir un seuil d'accessibilité}

Dans un processus d'augmentation de long terme de la vitesse des moyens de déplacements, la traduction concrète de ces performances dans les pratiques évolue par seuils. Ces seuils marquent l'apparition de nouvelles possibilités, pour la société et les individus, d'utiliser le temps et l'espace. Le chemin de fer réduira ainsi la France aux proportions d'un trajet de 24 heures aux alentours de 1870, à une époque où l'unification du marché national se verra considérablement renforcée. Aujourd'hui, l'enjeu est pour les individus, de pouvoir inscrire leurs déplacements dans une journée "normale" d'activité. L'offre de transport qui répond à cette exigence pour un coût économique supportable est alors assurée du succès.

A l'échelle urbaine, cette étape est depuis longtemps franchie, à l'aide des réseaux de transport en commun ou de l'automobile. Au niveau interurbain, dès que les distances s'allongent, il n'est pas toujours possible de réaliser un voyage dans la journée en conservant une plage de temps importante disponible à destination autrement qu'en recourant à l'avion. De ce point de vue, la mise en service du TGV entre Paris et Lyon, en offrant la possibilité d'allers-retours dans la journée, voire la demie journée, pour un tarif bien inférieur, a permis non seulement de capter une très large part de la clientèle aérienne, mais aussi de développer des comportements de mobilité privilégiant des déplacements plus fréquents et moins longs. La définition de ce seuil d'accessibilité combine donc les performances physiques et tarifaires des modes de transport en présence et les contraintes temporelles des usages.

La cause est entendue pour le cas lyonnais : le TGV a vraiment marqué une étape dans la facilité de relier Paris et Lyon (Müller, Papinutti et Reynaud, 1987). La situation de Nantes se rapproche beaucoup de celle de Lyon puisque le temps de parcours du TGV est identique. On note cependant que l'amélioration induite par le TGV est moins importante que dans le premier cas. Avec un temps de parcours de trois heures entre Paris et Bordeaux, le TGV ne fait qu'aborder le seuil d'accessibilité que nous décrivions. Il rend possible certains des comportements de mobilité évoqués, mais dans des conditions beaucoup moins bonnes que l'avion. La tarification élevée du TGV-A vient encore réduire l'avantage comparatif dont il peut bénéficier en 1993. Nous sommes sur cet exemple dans le même schéma explicatif qu'avec les deux villes précédentes, mais devant un phénomène très atténué.

La situation de Tours est en revanche radicalement différente. Tout d'abord, le train et la voiture sont les deux seuls modes en présence. Ensuite, ils rendent tout deux possible, dès 1989, la réalisation de déplacements vers Paris sans empiéter sur la journée de travail. Enfin, il faut tenir compte d'une part des aspects tarifaires déjà évoqués, d'autre part, des contraintes - particulièrement sensibles sur les courts trajets - que le mode de commercialisation du TGV (notamment l'obligation de réserver sa place) introduit. Dans ces conditions, il semble que le TGV a pu améliorer certains aspects de l'accessibilité ferroviaire entre Tours et Paris, mais qu'il n'a pas permis d'approcher un nouveau seuil. En outre, et de manière complémentaire, le TGV n'a pas non plus creusé d'écart déterminant entre l'offre ferroviaire et l'offre routière.

\subsection{Usages}

L'arrivée du TGV dans chacune des agglomérations étudiée ici a correspondu à une évolution importante des comportements de déplacement depuis ou vers Paris. Grâce aux enquêtes déjà 
mentionnées, ces évolutions ont été observées. Nous nous limitons ici à l'examen des déplacements à motif professionnel et nous présentons ces résultats ville par ville en commençant par Lyon en raison de son antériorité (3.3.1), puis Tours (3.3.2), Nantes (3.3.3) et enfin Bordeaux (3.3.4).

\subsubsection{Lyon en 85 : l'usage de la grande vitesse}

En 1980, la répartition modale du trafic d'affaires entre Paris et Lyon a pu être estimée à un tiers pour le train et deux tiers pour l'avion (la route, de toute façon marginale, n'a pas été prise en compte) (Bonnafous, Patier-Marque et Plassard, 1981). La clientèle des deux modes est à 90\% constituée de cadres qui effectuent une proportion déjà importante de déplacement en aller-retour sur la journée (près de 40\%). L'avion se distingue par le poids plus important de ce type de voyage. Concernant les motifs de déplacements, on voit apparaître deux grandes catégories : les motifs "marchands" (achats-ventes) et les motifs liés à des contacts internes à l'entreprise, au groupe ou à l'administration pour lequel travaille le voyageur enquêté. Les secteurs d'activité générant ces trafics sont enfin largement dépendants des caractéristiques du tissu économique lyonnais. On retrouve le poids important de l'industrie lourde mais aussi des activités tertiaires développées.

Entre 1980 et 1985, on estime que le trafic d'affaires s'est accru de près de 60\%. Le partage modal est devenu très favorable au chemin de fer (environ $85 \%$ du marché pour le TGV) (Bonnafous, 1987). On assiste dans le même temps à un raccourcissement de la durée des voyages (55\% d'allers-retours dans la journée) et à une augmentation corrélative de la fréquence de déplacement. En outre, les voyages effectués à plusieurs sont plus nombreux, ainsi que les déplacements par les membres de catégories professionnelles jusque là exclues de la mobilité d'affaires. Deux motifs semblent particulièrement porteurs de cette intensification des échanges : d'une part les achats-ventes de services (surtout des services aux entreprises), et d'autre part les "contacts internes à l'entreprise". Ces évolutions ne remettent pas fondamentalement en cause la hiérarchie établie entre Paris et Lyon : les Parisiens viennent davantage vendre qu'acheter et ce sont plutôt les Lyonnais qui vont rencontrer leurs collègues parisiens que l'inverse. Mais le déséquilibre ne semble pas non plus, d'après l'observation de la mobilité, se renforcer.

Globalement, on assiste à un rapprochement de Paris et Lyon que traduit l'adoption de comportements de déplacements de relative proximité. L'offre de transport à grande vitesse permet la diffusion de pratiques nouvelles de déplacement, mais aussi, semble-t-il, de modes d'organisation plus intégré entre les implantations lyonnaise et parisienne d'une même entreprise.

\subsubsection{Tours en 93 : Paris s'éloigne malgré le TGV}

En 1989, le chemin de fer bénéficie sur la relation Paris-Tours d'une position confortable : il assure près des deux tiers des déplacements pour motif professionnel. La faible distance entre les deux agglomérations induit un trafic de proximité. On constate ainsi que $70 \%$ des déplacements sont des allers-retours effectués dans la journée. L'importance des déplacements motivés par une réunion interne à l'entreprise (le groupe ou l'administration) pour laquelle travaille la personne qui se déplace est le signe que l'organisation productive intègre elle aussi la possibilité de relations de voisinage.

Partant de cette situation très favorable, les observations de 1993 marquent une très nette dégradation. Globalement, le volume de déplacements d'affaires entre Paris et Tours est en diminution importante. Cette diminution est concentrée sur le trafic ferroviaire qui baisse, sur les journées observées, de 40\%. Elle affecte également de manière privilégiée les déplacements liés à des contacts internes à l'entreprise (au groupe ou à l'administration) d'origine du voyageur. Enfin, le nombre d'allers-retours effectués dans la journée décroît un peu plus fortement que le trafic global. Tout se passe donc comme si Tours et Paris s'étaient éloignées l'une de l'autre entre 1989 et 1993.

On peut encore affiner les observations pour noter que seuls deux secteurs d'activité réussissent à peu près à maintenir les flux de trafic qu'ils génèrent entre Paris et Tours : celui des services non-marchands, et surtout celui des "études-conseils-assistance". De même, l'évolution la moins défavorable est le fait des cadres administratifs et commerciaux ainsi que des professions 
libérales. Enfin, on note avec intérêt un développement très important des allers-retours inscrits dans la demi-journée. Mais, confidentielle en 1989, cette pratique demeure marginale en 1993. Son extension montre néanmoins que les performances techniques du TGV entre Paris et Tours laissent ouvertes des possibilités nouvelles de comportements de mobilité. Le fait que ces possibilités ne répondent pas à la nécessité de nouveaux modes d’organisation renvoie pêle-mêle à la conjoncture dégradée, aux caractéristiques des échanges entre Paris et Tours, à leur relative proximité qui laisse un rôle important à la voiture et aux aspects tarifaires et commerciaux de l'offre ferroviaire. Seule la prise en compte de la totalité du cadre socio-technique dans lequel est venue s'inscrire la modification de l'offre de transport entre Paris et Tours permet de comprendre pourquoi ces deux agglomérations s'éloignent entre 1989 et 1993 alors même que le TGV a réduit de 46\% le temps de trajet ferroviaire entre elles.

\subsubsection{Nantes en 93 : la confirmation des résultats de Lyon}

La relation Paris-Nantes est, en 1989, l'une des rares sur laquelle le trafic d'affaires se répartit de manière plutôt équilibrée entre les trois modes de transport en présence : la voiture (37\%), le train (36\%) et l'avion (27\%). Cependant, la spécificité de chaque mode est importante. L'avion est ainsi le moyen de transport préférentiel des Parisiens et surtout des cadres qui représentent environ $80 \%$ de sa clientèle. Il est utilisé pour les séjours courts (plus de $60 \%$ d'allers-retours dans la journée), pour des activités liées au fonctionnement internes des entreprises. L'usage de la voiture est le fait de salariés de secteurs industriels se déplaçant avant tout pour des motifs liés à l'achat ou la vente de produits ou de services. Malgré la distance, presque la moitié des déplacements routiers sont des allers-retours effectués dans la journée. Le train est en revanche le moyen des voyages de plusieurs jours. C'est aussi celui des Nantais (85\% de la clientèle) et des salariés de la fonction publique.

Ce paysage très traditionnel est bouleversé en 1993. En premier lieu, il faut souligner la forte croissance du volume de déplacements d'affaires entre Paris et Nantes (+40\%), malgré une crise économique à l'évidence moins sensible ici au moment de l'enquête. En second lieu, le partage du trafic entre les modes a fortement évolué puisque l'avion est devenu marginal alors que le trafic ferroviaire a doublé, au minimum. On retrouve une configuration qui rappelle fortement le cas lyonnais, à ceci près que la voiture assure encore un bon quart des flux totaux.

L'impact quantitatif du TGV sur le trafic se retrouve lorsqu'on examine les caractéristiques des voyageurs et de leurs déplacements. Le trafic routier, tout d'abord, se développe sur les voyages de plusieurs jours, mais diminue sur les allers-retours d'une journée tout en restant lié aux motifs achats-ventes. Le trafic ferroviaire surtout explose véritablement sur les voyages de très courte durée (A-R dans la demi-journée : x10, A-R dans la journée : x4,5) et connaît une croissance encore vigoureuse concernant les séjours de $2-3$ jours. Il voit se multiplier la pratique du déplacement réalisé à plusieurs. Il capte une part importante de la clientèle du secteur tertiaire en général, et de celui des "études-conseil-assistance" en particulier. Il se développe désormais sur les motifs "achats-ventes" comme sur les motifs "contacts internes à l'entreprise".

Par rapport à la situation constatée à Lyon en 1985, la principale différence tient à ce que les Parisiens semblent réagir plus fortement à l'offre nouvelle que les Nantais. Il faut d'une part y voir un effet d'apprentissage ; les Parisiens ayant l'expérience du réseau sud-est ont plus rapidement su profiter du TGV. Il faut d'autre part y déceler un effet de conjoncture et de domination relative de l'économie parisienne : avec la crise, le motif "achat", plutôt provincial, s'effondre alors que les voyages liés à la vente, majoritairement parisiens, se maintiennent. Dans ce contexte, les Parisiens réagissent plus parce qu'ils maintiennent mieux leur mobilité. Enfin, on ne constate aucun phénomène de "démocratisation". Au contraire, la mobilité des personnels subalternes diminue alors que celle des cadres augmente. 


\subsubsection{Bordeaux en 93 : la grande vitesse en attente}

En 1989, l'avion occupe déjà plus de la moitié du marché des déplacements d'affaires entre Paris et Bordeaux et, compte tenu de la distance, la voiture n'apparaît plus que de manière marginale. On retrouve la même spécialisation de l'avion qu'à Nantes sur les allers-retours dans la journée et sur une clientèle de cadres. De même, le train est plutôt dévolu aux séjours plus longs et est utilisé de manière préférentielle par les salariés de la fonction publique.

En 1993, la situation apparaît en revanche totalement différente de l'autre métropole atlantique. Le volume de déplacements d'affaires mesuré lors de l'enquêtes est en baisse sévère ($25 \%)$. Cette baisse, déjà sensible sur le trafic aérien, est très marquée sur le trafic ferroviaire. Il semble que l'on observe en premier lieu ici une réduction généralisée de la mobilité entre Paris et Bordeaux qui concerne l'ensemble des modes et touche davantage les voyages effectués à plusieurs que les déplacements de personnes seules. Toutes les professions réduisent leur mobilité, mais les cadres accroissent leur part relative. La baisse est assez uniformément répartie selon les secteurs d'activité mais épargne néanmoins celui des "études-conseils-assistance". Les déplacements motivés par un achat sont en très forte diminution, ceux liés à des "contacts internes à l'entreprise" se réduisent. Seuls les ventes continuent à susciter autant de trafic.

La différence d'évolution des trafics aériens et ferroviaires s'explique en grande partie par leur positionnement initial, l'avion bénéficiant globalement d'une meilleure résistance de ses segments de marché. Mais on constate peu de glissement de clientèle d'un mode vers l'autre. Le seul mouvement significatif concerne, pour partie seulement, le segment des allers-retours dans la journée pour lequel le TGV semble trouver une relative efficacité face à l'avion.

On voit bien, au regard de la situation bordelaise, que la réalisation des potentialités d'une offre de transport à grande vitesse est aussi largement déterminée par des éléments a priori étrangers à la sphère des transports. La crise de 1993 est effet déterminante. La capacité du TGV à reprendre des parts de marchés à l'avion est pourtant réelle sur cette relation. Elle s'exprimera après 1993, lorsque la crise s'atténuera, lorsque la SNCF assouplira sa politique tarifaire, mais aussi, et paradoxalement, lorsque la concurrence aérienne se développera par suite de libéralisation.

\section{CONCLUSION}

Le premier point qui apparaît à l'examen des quatre exemples qui viennent d'être présentés concerne la diversité des évolutions de trafic auxquelles conduit la modification de l'offre de transport. Même en ne prenant en compte que les volumes de passagers, le TGV n'a à l'évidence pas un effet systématique de dynamisation des échanges avec Paris. Si l'on s'intéresse en outre au contenu de ces flux, la diversité des trajectoires se renforce encore. Le mythe des effets automatiques supporte bien mal la confrontation avec la réalité.

Le deuxième point que nous voudrions souligner revient en quelque sorte à mieux définir ce que nous observons à travers les enquêtes sur lesquelles nous nous sommes appuyés. A un premier niveau, lorsque l'on analyse les données questionnaires par questionnaires, on visualise des déplacements. A ce second niveau, et malgré les difficultés mentionnées en 1.2, l'agrégation statistique de ces observations unitaires fournit une certaine représentation des comportements de mobilité qui se développent sur la relation considérée. A un troisième niveau, c'est une image de la pratique de l'espace et du temps telle qu'elle ressort des activités économiques qui peut être construite.

Lorsque l'on traite de problématiques telles que transport et développement régional ou encore transport et formes urbaines, c'est, plus ou moins implicitement, à ce troisième niveau que l'on se réfère. Dès lors, on voit bien que les modifications de l'offre de transport constituent l'un des éléments à mobiliser pour l'analyse, mais qu'elles ne sauraient suffire. De même, on voit bien se dilater l'horizon temporel à prendre en compte, s'enchevêtrer le court et le long terme. C'est la nature même des problématiques abordées, le caractère global du regard à porter, qui donne une pertinence à la grille de lecture suggérée par les travaux de Patrice FLICHY. 
La mise en oeuvre qui en a été faite ici aura pu paraître quelque peu laborieuse par son systématisme. Elle permet d'insister sur la nécessité de croiser la description de phénomènes d'échelle macro-sociale ou macro-économique avec des approches plus fines et plus précises de la réalité. Elle avait aussi pour objet de prouver par l'exemple qu'il est possible de donner un contenu à l'environnement socio-économique, au cadre fonctionnel et au cadre d'usage qui constituent notre outil d'analyse. En revanche, les limites imparties à un article de revue ne permettaient guère plus que de suggérer la manière dont les différents éléments d'analyse qui ont pu être évoqués peuvent s'articuler. Il est pourtant essentiel d'insister sur ce qui relie ces éléments, de donner une structure d'ensemble à la représentation qui a ainsi été dessinée point par point. On peut faire confiance au lecteur qui, initié par les oeuvres impressionnistes, saura prendre le recul nécessaire. On peut aussi s’appuyer, et ce n’est pas son moindre intérêt, sur la grille de lecture proposée par Patrice FLICHY pour articuler cadre fonctionnel et cadre d'usage. Quoiqu'il en soit, cette mise en perspective reste, pour l'essentiel, à opérer.

\section{BIBLIOGRAPHIE :}

Arthur, W. Brian, 1995, La localisation en grappe dans la 'Silicon Valley' : à quel moment les rendements croissants conduisent-ils à une situation de monopole ? In Rallet, Alain, Torre, André, Economie industrielle et économie spatiale, Economica, coll.Bibliothèque de science régionale, Paris, pp.297-316.

Bonnafous, Alain, 1987, The régional impact of the TGV, Transportation, Vol. 14, n 2, pp.127137.

Bonnafous, Alain, Patier-Marque, Danièle et Plassard, François, 1981, Les déplacements d'affaires Paris-Rhône-Alpes, Laboratoire d'Economie des Transports, Lyon, , 88 p. .

Boyer, Robert, 1992, Les alternatives au fordisme - Des années 1980 au XXIème siècle. In Benko, Georges, Lipietz, Alain, Les régions qui gagnent - Districts et réseaux : les nouveaux paradigmes de la géographie économique, Presses Universitaires de France, coll.Economie en liberté, Paris, pp. 189-223.

Braudel, Fernand, 1979, Civilisation matérielle, Economie et Capitalisme, XVème-XVIIIème siècle, Armand Colin, Paris, 3 tomes.

Breton, Philippe, 1992, L'utopie de la communication, La Découverte, Paris, 151 p.

Burmeister, Antje , Joignaux, Guy, 1997, Infrastructures de transport et territoires - Approches de quelques grands projets, L’Harmattan, coll. Emplois, Industrie, Territoire, Paris, 319 p.

Calmette, Marie-Françoise, 1994, Localisation des activités et intégration européenne, Revue d'Economie Régionale et Urbaine, $\mathrm{n}^{\circ}$ 5, pp.741-764.

CEMT, à paraître, La mobilité induite par les infrastructures, Conférence Européenne des Ministres des Transports, coll. Table ronde 105.

Claisse, Gérard , Duchier, Didier, 1995, Des observatoires d'effets TGV : réflexions méthodologiques, Villes et TGV - Actes des 6èmes entretiens Jacques Cartier, Laboratoire d'Economie des Transports, coll. Etudes \& Recherches, Lyon, pp.219-238.

Cointet-Pinel, Odile , Plassard, François, 1986, Les effets socio-économiques du TGV en Bourgogne et Rhône-Alpes, Document de synthèse, DATAR, INRETS, OEST, SNCF.

Damette, Félix, 1994, La France en ville, La documentation française, DATAR, coll. Informations et analyses, Paris, $271 \mathrm{p}$.

Durand, Jean-Pierre, 1991, Travail contre technologie. In Durand, Jean-Pierre, Merrien, FrançoisXavier, Sortie de siècle - La France en mutation, Vigot, Paris, pp. 33-75. 
Flichy, Patrice, 1995, L'innovation technique - Récents développements en sciences sociales - Vers une nouvelle théorie de l'innovation, La Découverte, coll. Sciences et société, Paris, 250 p.

Klein, Olivier, 1995, Du rôle des villes dans la constitution du réseau à grande vitesse : entre nécessité et valorisation, Villes et TGV - Actes des 6èmes entretiens Jacques Cartier, Laboratoire d'Economie des Transports, coll. Etudes \& Recherches, Lyon, pp.267-274.

Klein, Olivier , Claisse, Gérard, 1997, Le TGV-Atlantique : entre récession et concurrence, Laboratoire d'Economie des Transports, coll. Etudes \& Recherches, Lyon, 163 p.

Krugman, Paul, 1991, Geography and trade, MIT Press, Cambridge, 142 p.

Mazier, Jacques, Basle, Maurice et Vidal, Jean-François, 1993, Quand les crises durent..., Economica, Paris.

Müller, Michel, Papinutti, Marc et Reynaud, Christian, 1987, Six ans de diffusion des effets du TGV sud-est, Les cahiers scientifiques du transport, $\mathrm{n}^{\circ}$ 15-16, pp.127-150.

Offner, Jean-Marc, 1980, L'évaluation des investissements de transport : effets ou congruence ?, Etudes de suivi et processus de décision - troisième journée du GRECO du C.N.R.S. Transport et Espace, INRETS, Arcueil, pp. 90-99.

Orfeuil, Jean-Pierre, 1994, Je suis l'automobile, Editions de l'aube, coll. monde en cours, La Tour d'Aigues, 95 p.

Plassard, François, 1976, Les autoroutes et le développement régional, Economica, Presses Universitaires de Lyon, Paris, Lyon, 341 p.

Plassard, François, 1989, Interconnexions et nouvelles polarités, Actes du colloque "Les couloirs Rhin-Rhône dans l'espace européen", Octobre 1989, Laboratoire d'Economie des Transports, Lyon, pp. 291-307.

Plassard, François , Routhier, Jean-Louis, 1986, Enquête TGV-Déplacements d'affaires, Transports, Laboratoire d'Economie des, Lyon, , 16p.+annexes .

Pumain, Denise, 1982, Chemin de fer et croissance urbaine en France au XIXe siècle, Annales de géographie, Vol. XCI, n 507, sept.-oct., pp.529-550.

Ragni, Ludovic, 1995, Systèmes localisés de production et modèles d'évolution dynamiques. Enjeux et limites des travaux de Brian Arthur et Paul Krugman. In Rallet, Alain, Torre, André, Economie industrielle et économie spatiale, Economica, coll.Bibliothèque de science régionale, Paris, pp. 335-357.

Revue d'Histoire des Chemins de Fer, 1995, Les très grandes vitesses ferroviaires en France, $n^{\circ} 12-13$.

Rosanvallon, Pierre, 1989, Le libéralisme économique - Histoire de l'idée de marché, Seuil, coll. Point Politique, Paris, 287 p.

Sociologie du travail, 1993, Vol. XXXV, $\mathrm{n}^{\circ} 1$.

Studény, Christophe, 1990, Le vertige de la vitesse, l'accélération de la France (1830-1940), Thèse de Doctorat, Ecole des Hautes Etudes en Sciences Sociales, Paris, 1075p. + annexes.

Toutain, Jean-Claude, 1967, Les transports en France de 1830 à 1965, Economie et Société-Les cahiers de l'I.S.E.A., $\mathrm{n}^{\circ}$ 8, septembre-octobre, p. 277.

Veltz, Pierre, 1993, D'une géographie des coûts à une géographie de l'organisation; Quelques thèses sur l'évolution des rapports entreprises/territoires, Revue économique, $\mathrm{n}^{\circ} 4$, juillet, pp. 671-684. 